\title{
Flowing afterglow apparatus for the study of ion-molecule reactions at high temperatures
}

\author{
Peter M. Hierl, ${ }^{a)}$ Jeffrey F. Friedman, ${ }^{\text {b) }}$ Thomas M. Miller, ${ }^{c)}$ I. Dotan, ${ }^{d)}$ \\ Melani Menendez-Barreto, ${ }^{\text {e) }}$ John V. Seeley, ${ }^{\text {f) }}$ John S. Williamson, ${ }^{\text {,) }}$ Fred Dale, \\ Paul L. Mundis, ${ }^{\text {g) }}$ Robert A. Morris, John F. Paulson, and A. A. Viggiano ${ }^{\text {h) }}$ \\ Phillips Laboratory, Geophysics Directorate, Ionospheric Effects Division (GPID), \\ Hanscom Air Force Base, Massachusetts 01731-3010
}

(Received 3 January 1996; accepted for publication 26 February 1996)

\begin{abstract}
We describe two versions of a high temperature flowing afterglow apparatus. With a stainless steel flow tube wrapped with heating tape we have obtained data over the range 300-1300 K. In a version with a ceramic flow tube in a commercial furnace we have obtained data over the range 300-1600 $\mathrm{K}$. The ceramic version is designed to take data up to $1800 \mathrm{~K}$, but we have encountered experimental problems at the upper temperature range. The design modifications to a standard flowing afterglow needed to make measurements at elevated temperatures are described in detail, as are problems associated with operating at elevated temperatures. Samples of data are given. (C) 1996 American Institute of Physics. [S0034-6748(96)01006-4]
\end{abstract}

\section{INTRODUCTION}

The need for understanding the chemical behavior of plasmas has spawned the development of techniques for studying ion-molecule reactions at elevated temperatures. High temperature ion chemistry occurs in the ionosphere, in combustion situations, in plasma chemical reactors, and in plasmas associated with atmospheric reentry and hypersonic flight. In the case of atmospheric reentry, e.g., a plasma may envelope the vehicle and interfere with radio communication. Computer models are used to describe the complicated chemistry in these plasma situations, such as reentry, but the models require accurate and meaningful kinetics data in order to describe and predict the plasma properties. While there is a wealth of ion chemical kinetics data available in the literature, very little covers temperatures above $300 \mathrm{~K}$, and such data above $900 \mathrm{~K}$ are essentially nonexistent. High temperature kinetics data are often required for modeling plasmas at elevated temperatures because extrapolation of low temperature data is unreliable.

One way to circumvent the temperature limitation has been to study reactions at elevated ion energy in drift tubes and ion beam apparatuses. ${ }^{1}$ These techniques have been highly successful and produced an abundance of data on many systems. However, results from these techniques offer only a guide for predictions on the behavior of reaction rate constants and product branching fractions at elevated temperatures because it is the translational energy (and perhaps

\footnotetext{
${ }^{a)}$ NRC Senior Research Program. Permanent address: Department of Chemistry, University of Kansas, Lawrence, KS 66045-0046.

b) AFOSR Summer Faculty Research participant, 1992 and 1993. Permanent address: Department of Physics, University of Puerto Rico, Mayaquez, PR 00680.

${ }^{c}$ Under contract to Visidyne, Inc., Burlington, MA.

${ }^{d)}$ AFOSR Summer Faculty Research participant, 1995. Permanent address: The Open University of Israel, 16 Klausner St., Ramat Aviv, Tel Aviv, Israel.

e) AFOSR Summer Graduate Student Research participant, 1992 and 1993.

${ }^{\text {f) }}$ Air Force Geophysics Scholar.

${ }^{g}$ Under contract to Wentworth Institute, Boston, MA.

${ }^{\text {h) }}$ Author to whom correspondence should be addressed.
}

the internal energy of the ion) that is raised in ion beam and drift tube experiments. It has been shown that temperature and kinetic energy often have differing effects on reactivity. We have studied many systems as functions of both temperature and kinetic energy and have observed a variety of behaviors, e.g., sometimes increasing temperature increases reactivity while kinetic energy decreases reactivity. ${ }^{2}$ In other cases, increasing temperature decreases reactivity more effectively than does kinetic energy. ${ }^{3}$ Thus in some cases kinetic energy dependences may be misleading indicators of temperature dependences, and clearly there is a need for measurements at true temperatures.

While the kinetics of most ion-molecule reactions have been measured only at room temperature, there has been a considerable amount of work in studying temperature dependences of ion molecule reactions over the range between 80-600 K. ${ }^{4}$ These temperature dependence studies have shown interesting features including the important observation that rate constants for ion-molecule reactions frequently decrease with increasing temperature, presumably because the lifetime of the reaction complex decreases with collision energy.

There have been only two studies outside our laboratory where rate constants of ion-molecule reactions were measured above $600 \mathrm{~K}$. The first set of measurements was made at the NOAA Aeronomy Laboratory in 1974 using a flowing afterglow apparatus designed to operate over the temperature range 80-900 K. ${ }^{5}$ A total of 9 reactions were studied up to $900 \mathrm{~K}$, all involving simple systems. On reflecting back on these measurements, Ferguson has stated "This was painfully laborious. The materials problems at $900 \mathrm{~K}$ are horrendous, and the measured rate constants at $900 \mathrm{~K}$ had much larger uncertainties than the room temperature measurements." ${ }^{\prime 6}$ The second experiment was carried out at the University of Pittsburgh in a static drift tube designed to operate up to $1500 \mathrm{~K}^{7}$ Measurements were made up to 930 $\mathrm{K}$ on two reactions that had been previously studied by the NOAA group. Measurements were not made above $930 \mathrm{~K}$ due to thermionic emission of alkali ions from the drift tube 

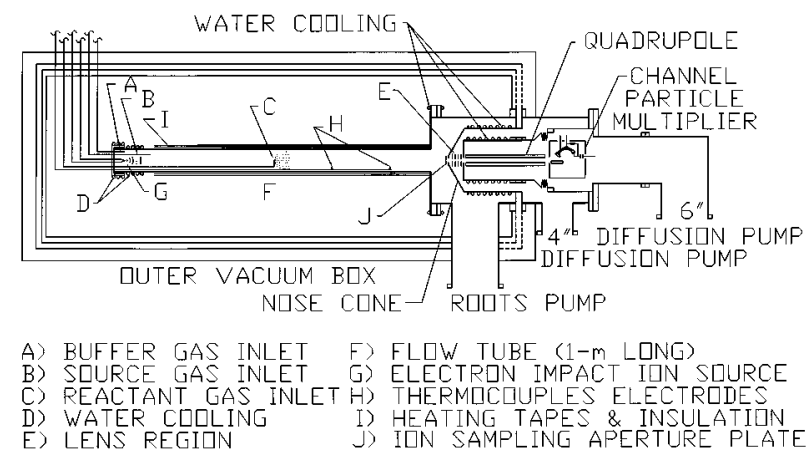

FIG. 1. Schematic of the stainless steel version of the high temperature flowing afterglow.

walls. For reactions with $\mathrm{O}_{2}$, an upper limit of $700 \mathrm{~K}$ was reached due to reaction of $\mathrm{O}_{2}$ with the tantalum walls.

Fontijn has succeeded in building neutral kinetics instruments capable of measurements in the vicinity of $1900 \mathrm{~K}^{8}$ Also Knudsen cell mass spectrometers have been operated over $2000 \mathrm{~K} .{ }^{9,10}$ McFadden and colleagues have studied electron attachment in a flow tube at temperatures up to 800 $\mathrm{K}$ although the instrument is designed to study reactions up to $1300 \mathrm{~K}^{11,12}$ These experiments have encouraged us to conclude that it is possible to study ion-molecule reactions above $1000 \mathrm{~K}$.

In this article we describe a flowing afterglow system designed to operate at temperatures up to $1800 \mathrm{~K}$. The system was built in two stages. The first involved a stainless steel flow tube wrapped with heating tape and capable of studying reactivity up to $1300 \mathrm{~K}$. One paper has been published with data from this system. ${ }^{13}$ More recently we have replaced the stainless steel/heating tape system with a ceramic flow tube mounted in a commercial furnace. At present we have data up to $1600 \mathrm{~K}$ and hope to be able to study reactivity at temperatures of $1800 \mathrm{~K}$ in the near future. This article will cover the modifications to a conventional flowing afterglow necessary to make measurements at these temperatures and to present some of the first data at temperatures up to $1600 \mathrm{~K}$.

\section{EXPERIMENT}

Flowing afterglows have been used to study ionmolecule reactions since the early $1960 \mathrm{~s} .{ }^{14}$ The technique is well developed and has been thoroughly described in the literature. Two reviews are particularly noteworthy, one by Ferguson et al. ${ }^{15}$ that describes the principles and mathematics involved and one by Graul and Squires ${ }^{16}$ that describes the developmental history of the technique. Here we will concentrate on the aspects of the technique pertinent to making measurements at high temperature.

Our first attempt at making measurements at high temperature involved a stainless steel flow tube wrapped with heating tapes. We have replaced this system with an alumina flow tube and commercial furnace. ${ }^{17}$ These two versions of the experiment are similar in many aspects and will be described together with the differences noted. They are shown schematically in Figs. 1 and 2, respectively.

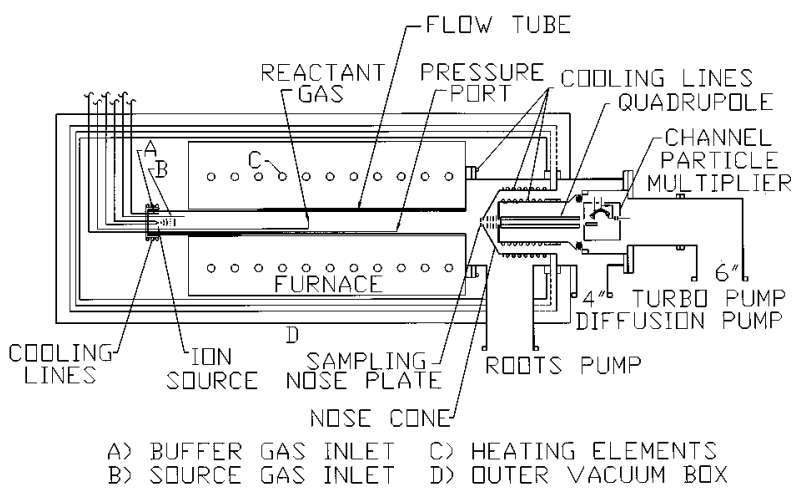

FIG. 2. Schematic of the ceramic version of the high temperature flowing afterglow.

Both versions of the instrument are fundamentally standard flowing afterglows. A buffer gas, usually helium, enters the flow tube (7.06 i.d., $106 \mathrm{~cm}$ long) at the upstream end. An ion source is located as far upstream as practical. Source gas can be added just upstream or downstream of the ion source. The ions are carried downstream by the buffer and past an inlet for the reactant neutral. At the downstream end of the flow tube the ions are sampled through a truncated nose cone with a sampling orifice $0.2 \mathrm{~mm}$ in radius. The ions pass through a differentially pumped lens region and enter a quadrupole mass filter. The ions are detected by a particle multiplier. The bulk of the buffer gas is pumped away by a Roots pump.

Reaction rate constants are measured by following the change in primary ion signal as a function of added neutral reactant flow rate. The rate constant is given by

$$
k=1 /\left([\mathrm{B}]^{*} \tau\right)^{*} \ln \left(\left[\mathrm{A}_{0}^{ \pm}\right] /\left[\mathrm{A}^{ \pm}\right]\right),
$$

where $k$ is the rate constant, $\tau$ is the reaction time, [B] is reactant neutral concentration, $\left[\mathrm{A}_{0}^{ \pm}\right]$is the primary ion concentration in the absence of reactant neutral, and $\left[\mathrm{A}^{ \pm}\right]$is the primary ion concentration. The reaction time is the reaction distance divided by the buffer velocity multiplied by a correction factor that accounts for the fact that both the ion velocity and concentration distributions are a maximum along the axis of the flow tube. A typical value for the correction factor is 1.6. The measurement of this correction factor will be discussed in detail later in the article. The buffer velocity is obtained from the mass flow rate of the buffer, the flow tube cross section, temperature, and pressure in the normal manner. ${ }^{15}$ In all the above aspects the high temperature flowing afterglow (HTFA) is similar to a conventional flowing afterglow.

While the basic principles of the HTFA are the same as any flowing afterglow, the extreme temperatures result in several modifications to normal designs. Besides the obvious need for a large heat source, the main design problems arise from the need for gas and electrical feedthroughs into the ceramic flow tube. In the HTFA, all feedthroughs enter through a cooled endcap (based on a design by the furnace manufacture ${ }^{17}$ ) at the upstream end of the flow tube. Figure 3 shows a three-dimensional drawing of the endcap with all the feedthroughs labeled. All lines attached to the endcap are 


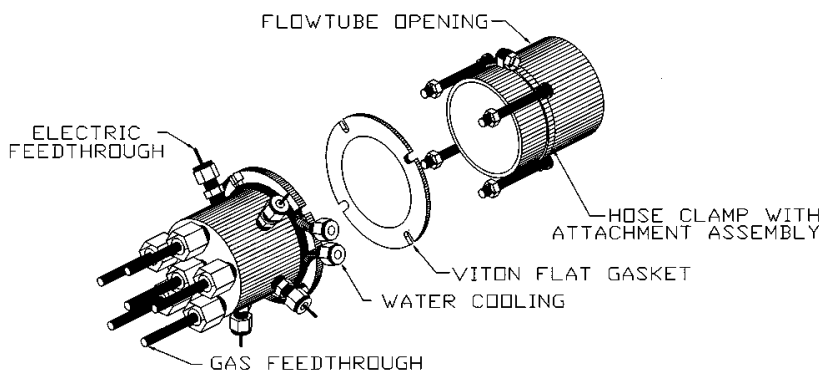

FIG. 3. Schematic of the endcap.

made from flexible stainless steel hose to allow for thermal expansion $(1.6 \mathrm{~cm}$ at $1200 \mathrm{~K})$ of the flow tube. The heated portion of the flow tube starts $\sim 10 \mathrm{~cm}$ downstream of the endcap flange. The endcap is water cooled and therefore conventional gas and electrical feedthroughs can be used. The i.d. of the endcap is the same as that of the flow tube. The o.d. of the endcap flange is larger than the o.d. of the flow tube to accommodate screws which pull the flange against a flat Viton gasket at the end of the flow tube. Connection to the ceramic flow tube is made with a stainless steel hose clamp into which four screw holders are inserted, as shown in Fig. 3. Four screws extend from the screw holders to slots in the endcap flange and are held there with wingnuts. This design was used for the stainless steel flow tube as well (rather than a welded fitting) as a prototype for the ceramic system. The cooled endcap in close proximity to the hot flow tube creates a temperature gradient in the upstream region of the flow tube of $\sim 100 \mathrm{~K} / \mathrm{cm}$ at the highest temperatures used. We have not experienced problems with this large gradient although one may expect problems with the ceramic cracking in the future.

\section{STAINLESS STEEL FLOW TUBE $(300-1300 \mathrm{~K})$}

Our first experiments were made with a stainless steel flow tube wrapped with heating tapes covered with Samox insulation. ${ }^{18}$ The heating tapes were wired in four zones and rated by the manufacturer to $1070 \mathrm{~K}$ and $5 \mathrm{~A}$ of current. We found the current limit more stringent than the temperature limit if care was taken not to disturb the tapes. Above 1070 $\mathrm{K}$ the Samox insulation on the heating wire crystallized. Left in place, the tapes could be operated indefinitely. Moving the tapes caused the crystallized insulation to crack. With the heating tapes alone we could achieve a temperature of only $800 \mathrm{~K}$, but the addition of several layers of loosely wrapped zirconia felt raised the temperature limit to $1300 \mathrm{~K}$.

Pressure was measured by inserting a capped stainless steel $1 / 4$ in. o.d. tube at the bottom of the flow tube, with a hole drilled in the $1 / 4 \mathrm{in}$. tube perpendicular to the flow. The pressure port was connected to a capacitance manometer. The connecting tube was large compared to the mean free path, and the pressure at the manometer was the same as that in the flow tube. ${ }^{19}$ Ion source gas entered at either the endcap or just downstream of the ion source. The latter inlet was a short piece of stainless steel $1 / 4$ in. o.d. tubing. The reactant neutral inlet was also a stainless steel 1/4 in. o.d. tube resting on the bottom of the flow tube and bent so that the end of the tube terminated in the center of the flow tube. The reaction distance was adjustable by moving the position of the tube in the endcap feedthrough.

Two thermocouples inside the flow tube were used to measure the temperature in the reaction zone. The thermocouples were run inside a 1/4 in. o.d. stainless steel tube into which a four-holed alumina insert was placed. The tube rested along the bottom of the flow tube. The $0.013 \mathrm{~cm}$ diam thermocouple wires exited their housing through holes placed $30 \mathrm{~cm}$ apart roughly centered in the reaction region. The wires protruded $2-3 \mathrm{~cm}$ along the radius of the flow tube. The room-temperature end of the four-holed alumina tube was sealed with vacuum epoxy. Using this setup we found that the two internal temperature readings could be maintained to within $4 \mathrm{~K}$ by manually controlling four variable autotransformers that powered the heating tapes. The internal temperature readings agreed well with the external temperature readings. The internal thermocouples also served as pulsing electrodes for measuring the ion velocity and as crude Langmuir probes for measuring the electron or ion density.

The downstream end of the stainless steel flow tube truncated into a thin $(3 \mathrm{~mm})$ flange welded to the flow tube. The flange was considerably wider than the flow tube, $38 \mathrm{vs} 7 \mathrm{~cm}$ in diameter. The outside of the flange was water cooled and sealed to the vacuum chamber by a Viton O-ring. The thinness of the flange inhibited heat flow. The weld between this flange and the flow tube eventually cracked due to repeated deformation inevitable with the i.d. at $1200 \mathrm{~K}$ and the o.d. water cooled.

\section{CERAMIC FLOW TUBE (300-1800 K)}

In the ceramic flow tube version of the instrument a commercial furnace ${ }^{17}$ is placed inside the vacuum box. The furnace utilizes silicon carbide heating elements. At present, an alumina flow tube $(7.6 \mathrm{~cm}$ o.d., $7 \mathrm{~cm}$ i.d.) is placed in the center of the furnace. Other materials such as mullite or zirconia are alternatives which we have not yet tried. The furnace is made up of two short heating zones at each end and a long central zone. The temperature is controlled by three temperature controllers ${ }^{20}$ which automatically adjust the amount of current needed to maintain a constant temperature. The controllers keep the temperature constant to within $2 \mathrm{~K}$ as measured by thermocouples inserted into the furnace and abutting the flow tube. The total power consumption at maximum temperature is $13.5 \mathrm{~kW}$. The maximum temperature is rated at $1873 \mathrm{~K}$ but the manufacturer recommends a practical limit of $1800 \mathrm{~K}$ to prolong the life of the heating elements. The furnace weighs $500 \mathrm{lbs}$ which prohibits its easy movement. Since removal of the furnace is required for assembly and maintainence of the sampling plate and electrodes, we constructed a forklift to move the furnace. This enables the lens system to be taken apart and reassembled in a matter of hours.

The upstream end of the ceramic flow tube utilizes the same endcap as used with the stainless steel version. The downstream end of the furnace bolts to the water-cooled ion sampling chamber as shown in Fig. 2. The downstream end of the flow tube protrudes through a hole in a thin stainless 
steel plate at the end of the furnace by $5 \mathrm{~cm}$, with no vacuum seal. Leakage from this area is pumped from the vacuum box surrounding the flow tube and furnace by the Roots blower.

The inlets and pressure port in the ceramic tube are similar in principle to those used in the stainless steel flow tube. They are made of ceramic which causes some small engineering problems. For example, the right angle in the reactant inlet cannot be made by bending the tube. Instead, the inlet consists of three tubes connected by Graphi-Bond, a graphite based cement with a maximum operating temperature over $3000 \mathrm{~K}^{21}$ The reactant feedline is $1 / 4 \mathrm{in}$. o.d. where it passes through the endcap. Inside the flow tube the reactant inlet is $1 / 2$ in. o.d. The downstream end of the $1 / 2$ in. o.d. tube is sealed and a short piece of $1 / 4$ in. o.d. tube is cemented in place to carry the reactant gas to the axis of the flow tube. The opening of the reactant line on the axis of the flow tube defines the beginning of the ion-molecule reaction region. The ion sampling plate at the downstream end of the flow tube terminates the reaction region. The current reaction length is $53 \mathrm{~cm}$.

The ion source for both systems is an electron emitting filament biased against ground. A grid is placed near the filament. A light baffle $(1.9 \mathrm{~cm}$ o.d.) mounted on the end of the ion source assembly prevents UV light from the ion source from photoionizing source and/or reactant gases along the flow tube. The filament holder, grid, and baffle are supported on a rod emerging from the endcap. The source is kept upstream of the heated region. This prevents the ceramic insulators from becoming conducting at high temperature and the filament from being degraded. It also allows use of some metal components that could not tolerate the highest temperatures obtainable in the heated region.

The first surface exposed to the gas upon exiting the heated interaction region is an electrically insulated sampling plate attached to the nose cone. The plate is made of molybdenum. The plate is screwed to the nose cone and separated from it by a thin ring-shaped ceramic insulator that also acts as a seal between the flow tube gas (typically 1 Torr) and the ion lensing chamber (typically $10^{-4}$ Torr). This seal has cracked at times and has acquired a conducting coating at times. The nose cone is attached to the main chamber by a metal-to-metal seal (i.e., no gasket).

The nose cone and sampling plate were designed to minimize heat transfer so that the sampling plate stays as hot as possible; however, we have not measured the plate temperature. Minimizing heat transfer is accomplished by making the nose cone as thin as possible while maintaining structural stability. The ceramic electrical insulation between the nose plate and the nose cone also acts as an insulator for heat conduction.

After the nose plate a series of lenses focus the ions into a separately pumped chamber containing a quadrupole mass spectrometer and particle multiplier. Both the ion lensing chamber and the mass spectrometer chamber are watercooled because of conduction and radiation of heat from the flow tube region of the apparatus.

The experiment is computer controlled. The pressure, temperature, gas flows, and signal intensity are monitored by an Apple Macintosh computer equipped with a GW Instru-

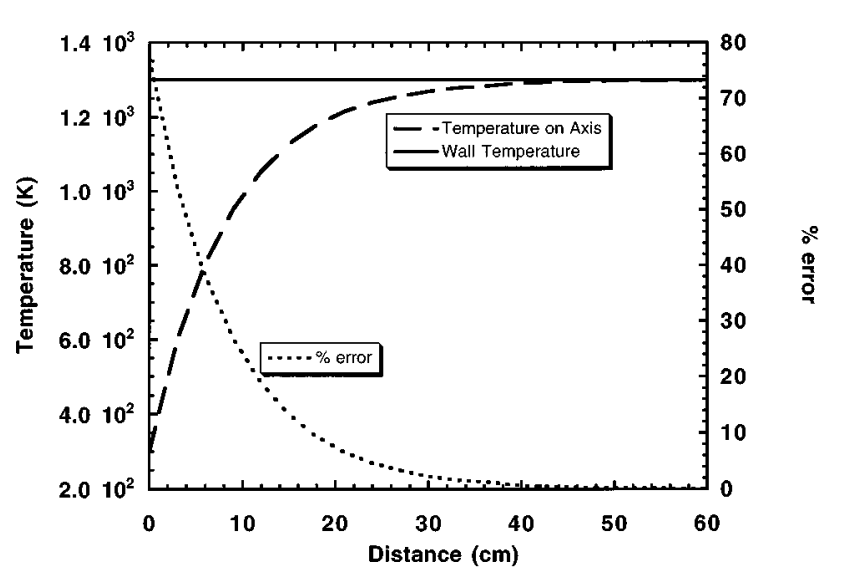

FIG. 4. Calculated on-axis temperature profile (large dashed line, left axis) and temperature error (small dashed line, right axis) as a function of distance. Also shown as a solid line is the wall temperature.

ments data acquisition card. A data run at a single temperature can be obtained within a few minutes. Changing the temperature by about $100 \mathrm{~K}$ takes about $15 \mathrm{~min}$, nominally. True temperature stability is checked by taking several runs at each temperature. Often the first two runs result in slightly different rate constants indicating that the flow tube temperature has not equilibrated. After a settling time of perhaps an additional $15 \mathrm{~min}$ the data are quite reproducible. An entire temperature run from 300 to $1600 \mathrm{~K}$ can be made in $10 \mathrm{~h}$ with rate constants taken every $100 \mathrm{~K}$.

The good agreement between external and internal temperature is expected from the entry length equation given by Gilbert, ${ }^{22}$

$$
\frac{T_{a}-T(z, 0)}{T_{a}-T_{0}}=\exp \left(-\frac{7.32 z}{N_{p} N_{r} R}\right),
$$

where $z$ is the distance from the upstream end of the heated region, $T_{a}, T(z, 0)$, and $T_{0}$ are the temperatures at the wall of the reactor, at position $z$ on the axis, and at the entrance on the axis of the flow tube, respectively. $N_{p}$ is the Prandtl number and $N_{r}$ is the Reynolds number. The Reynolds number is defined as ${ }^{23}$

$$
N_{r}=(2 R\langle u\rangle \rho) / \mu,
$$

where $\langle u\rangle$ is the average velocity of the buffer, $\rho$ is the density, $\mu$ is the viscosity. The Prandtl number is defined as $^{23}$

$$
N_{p}=C_{p} \mu / \kappa,
$$

where $C_{p}$ is the heat capacity, and $\kappa$ is the thermal conductivity.

Figure 4 shows a graph of distance along the flow tube vs axial temperature of the flow tube for typical conditions with the flow tube wall at $1300 \mathrm{~K}$. The calculated values of $N_{p}$ and $N_{r}$ used are 0.61 and 14.7, respectively. Also shown is the deviation of the axial temperature from the wall temperature in percent. The graph shows that the temperature is essentially uniform by a distance of approximately $30 \mathrm{~cm}$ into the furnace. The reactant neutral inlet is therefore kept downstream of this point. 


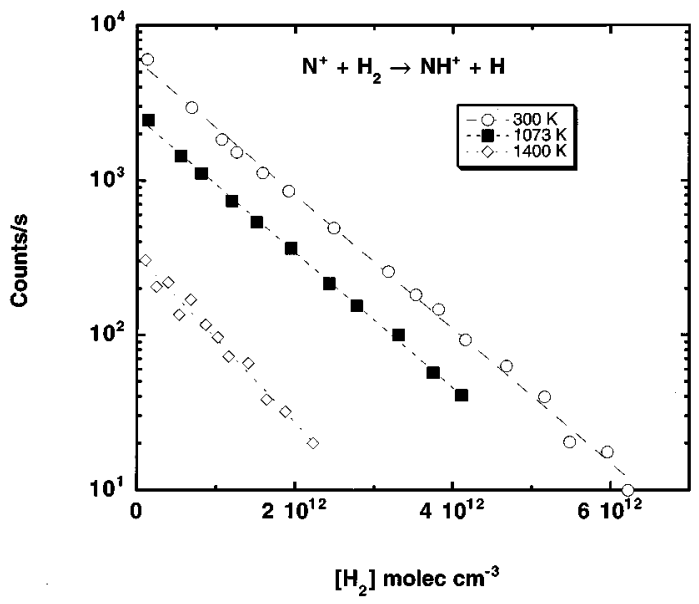

FIG. 5. Decay plots for the reaction of $\mathrm{N}^{+}$with $\mathrm{H}_{2}$ at 300,1073 , and $1400 \mathrm{~K}$.

An error limit on the rate constants is estimated as follows. The reproducibility of the rate constants is typically $<10 \%$. Sources of error include the above reproducibility, $\pm 10 \%$, and measurement of temperature, $\pm 2 \%$, pressure, $\pm 1 \%$, ion flight time, $\pm 5 \%$, an end correction related mainly to reactant gas mixing $( \pm 2 \mathrm{~cm}$ or $\pm 4 \%$ ), buffer gas flow rate (relative, $\pm 1 \%$, or absolute $\pm 2 \%$ ), and neutral reactant gas flow rate (relative, $\pm 3 \%$, or absolute, $\pm 15 \%$ ). Propagation of errors leads to a net relative uncertainty of $\pm 13 \%$ and a net absolute uncertainty of $\pm 19 \%$. The reaction rate constants measured with the HTFA apparatus will be given with relative and absolute uncertainties of $\pm 15 \%$ and $\pm 25 \%$, respectively, to account for the possibility of systematic errors not treated in the above analysis.

\section{RESULTS AND DISCUSSION}

Figure 5 shows a plot of the ion count rate vs reactant neutral concentration for the reaction of $\mathrm{N}^{+}$with $\mathrm{H}_{2}$ at several temperatures. The effect of diffusion on the ion signals is clearly seen in the $y$ axis intercepts, i.e., the signal decreases dramatically with increasing temperature. The room temperature signal is about a factor of 20 larger than the $1400 \mathrm{~K}$ signal. The plots show good linearity on a semilog plot over a factor of almost 3 orders of magnitude at room temperature and over one order of magnitude at high temperature. The good linearity indicates that the source conditions are such that production of the primary ion is complete by the time the neutral gas is added. As with any flowing afterglow experiment, choosing a source gas and setting the source conditions to achieve linearity in the data can be the most difficult part of the experiment. Nonlinearity was sometimes found and corrected simply by adjusting the source gas flow rate. Most often the nonlinearity resulted from too little source gas especially at high temperatures where the flow velocity is large and therefore a considerable amount of source gas was needed. One particularly interesting source of nonlinearity was found in $\mathrm{O}^{-}$reactions. When $\mathrm{N}_{2} \mathrm{O}$ was used as the source gas, nonlinearity became a problem at about $1000 \mathrm{~K}$. The source of the problem turned out to be the onset of thermal electron attachment to $\mathrm{N}_{2} \mathrm{O}$ producing a source of

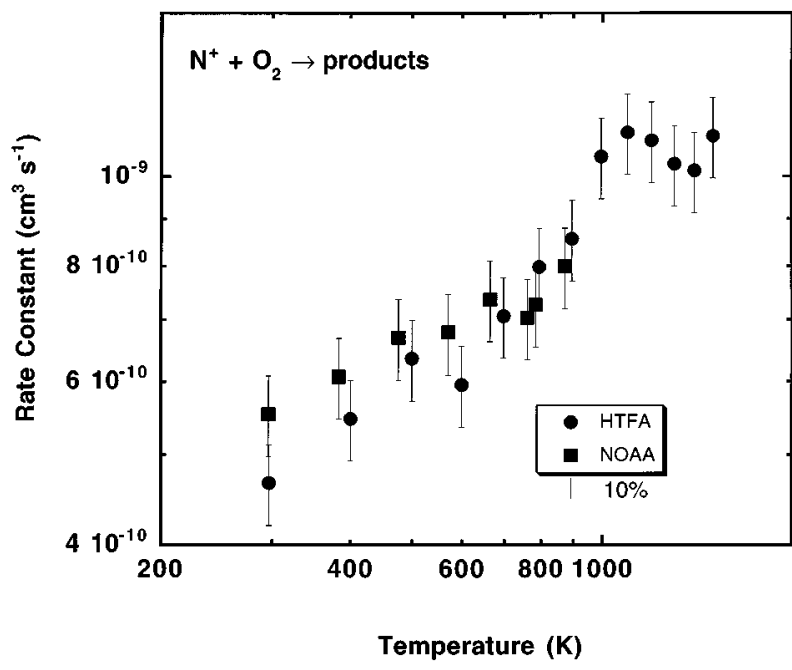

FIG. 6. Data for reaction of $\mathrm{N}^{+}$with $\mathrm{O}_{2}$ from 300 to $1500 \mathrm{~K}$. The NOAA data are taken from Lindinger et al. (Ref. 5) Error bars of $\pm 10 \%$ are shown for comparison. Quoted error bars are larger (see the text).

$\mathrm{O}^{-}$in the reaction region at high temperature. This particular problem was solved by using $\mathrm{CO}_{2}$ as a source gas upstream of the ion source and adding an electron scavenging gas in the downstream source inlet. Problems such as this show that one must be careful as new temperature regions are explored.

Figure 6 shows a plot of rate constants vs temperature for the reaction of $\mathrm{N}^{+}$with $\mathrm{O}_{2}$. Also shown are the data taken at high temperature by the NOAA group. ${ }^{5}$ Very good agreement is found in this and all reactions that both groups have measured. Good agreement is also found between data taken in the new system and those taken in our selected ion flow tube at temperatures up to $550 \mathrm{~K}^{13,24,25}$ and with the drift tube work of Chen et $\mathrm{ll}^{7}{ }^{7}$ We have published one paper on data taken in the stainless steel flow tube. ${ }^{13}$ Three reactions were reported: $\mathrm{O}^{-}$with $\mathrm{CO}, \mathrm{O}^{-}$with $\mathrm{NO}$ and $\mathrm{O}_{2}^{+}+\mathrm{CH}_{4}$. In all cases the agreement with previously published data was excellent.

The above shows that reliable data can be obtained to at least $1600 \mathrm{~K}$ with this apparatus. However, we have encountered several problems. One source of concern arises from positive alkali ion emission from both the flow tube and sampling noseplate. With the stainless steel tube experiments we found that most of the thermionic emission of alkali ions originated from the noseplate (as determined by shutting off the buffer flow and ion source and noting little or no change in the alkali ion intensity). The alkali ion problem was a significantly worse when a molybdenum nose plate was used. The dominant ion observed was $\mathrm{K}^{+}$, along with some $\mathrm{Na}^{+}$, and small amounts of $\mathrm{Rb}^{+}$and $\mathrm{Cs}^{+}$. At the highest temperatures reached thus far, the alkali ion intensity greatly exceeds intensities of the ions under study. So far, we have detected no error in measuring rate constants due to the alkalis. No neutrals should react with the alkali cations since the ionization potentials of the alkali atoms are so low. The main problem has been to resolve a moderate $\mathrm{Ar}^{+}$signal (40 Daltons) from a large $\mathrm{K}^{+}$signal (39 and 41 Daltons) with the mass spectrometer, while maintaining good signal intensity. 
Using a thermocouple as a Langmuir probe indicated that the alkali ion density was less than $10^{6} \mathrm{~cm}^{-3}$ at $1200 \mathrm{~K}$. At temperatures above $1600 \mathrm{~K}$, the concentrations may become large enough to cause problems. We are exploring the purchase of a higher purity flow tube and prolonged baking at high temperature to reduce the alkali concentrations.

Diffusive losses of the ions increase substantially with increasing temperature. The diffusion rate in our flowing afterglow Langmuir probe apparatus increases according to a $T^{1.5}$ dependence at constant pressure between 300 and 550 $\mathrm{K}^{26}$ Therefore, the ion signal decreases substantially at higher temperatures and it is loss of signal that has so far limited the upper temperature to about $1600 \mathrm{~K}$ in the HTFA. To help reduce this effect we flow more buffer gas at increasing temperature. The buffer flow at about $1000 \mathrm{~K}$ and higher is $29 \mathrm{slm}$, the maximum the buffer gas flow controller will deliver. This is a little more than twice the flow used at room temperature. (It is the expected diffusive losses at high temperatures that led us to construct a flowing afterglow reactor instead of a selected ion flow tube, in which the ion concentrations may be orders of magnitude smaller.) In the future we plan to try a larger flow rate and other buffers to help reduce the effect of diffusion.

Our experience with a reactant neutral finger inlet is that the end correction due to neutral mixing is very small $(\sim 2 \%)$. We have confirmed this by studying reactivity at two distances. No measurable difference was found.

The ion velocity is different than the bulk helium velocity since the ion concentration is peaked along the axis of the flow tube, coinciding with the maximum in the parabolic velocity profile of the buffer gas. If the ions remained on the flow tube axis during the entire flight time, the ratio $V_{\text {ion }} / V_{\text {buffer }}$ would be 2 , ignoring the few percent molecular slip of gas at the flow tube walls. Calculations which take into account random walk of ions within the radial ion distribution yield a ratio $R=1.6 .^{15,27}$ Measurements of $R$ in other flow tubes in our laboratory have given values as large as 1.8 at $300 \mathrm{~K}$ with a value of about 1.6 at $400 \mathrm{~K}$ and higher. ${ }^{28}$ With the HTFA apparatus, we have measured the ion velocity by replacing the reactant inlet with tubes to which two molydenum grids were attached. The limited number of feedthroughs in the endcap prevent us from leaving the pulsing grids in place permanently. By pulsing each of the grids separately and measuring the flight times, the velocity correction factor is derived. In the stainless steel system pulsing of the ion cloud was accomplished using the thermocouples as the pulsing electrodes. Between 300 and $1000 \mathrm{~K}$ we obtained values between 1.5 and 1.6. At temperatures approaching $1000 \mathrm{~K}$ the correction factor became very difficult to measure due to diffusion filling in the ion density hole. Above $1000 \mathrm{~K}$ the measurement of the velocity factor was not possible. We use a value of 1.6 at all temperatures to determine the ion velocity and hence the reaction time. We estimate this causes at most a $10 \%$ error in the rate constants.

Thermal decomposition of reactant gas is of course a potential problem. For now we are limited to stable gases not expected to decompose on the ceramic feedlines and flow tube surface. Since most of the wall collisions occur in the feed line due to its smaller diameter, building a cooled inlet may partially alleviate the problem. A residual gas analyzer in the detection region may help to diagnose this problem.

We have a separate selected ion flow tube (SIFT) apparatus that operates over the range from $80-550 \mathrm{~K}$. Good agreement in the overlapping temperature range means a combined operating range of $80-1600 \mathrm{~K}$. The good agreement stems in part from measuring all necessary quantities in both apparatuses, including the end correction for neutral mixing and the velocity correction factor.

In addition our SIFT apparatus contains a drift tube so that kinetic energy dependences can be measured. ${ }^{29}$ We have shown that comparing kinetic energy dependences at different temperatures allows one to obtain information on internal energy dependences. ${ }^{29}$ The higher temperature range now possible with the two apparatuses allows us to look for internal energy effects of high frequency vibrations not accessible in our SIFT studies alone. For instance we can excite vibrations of diatomic molecules such as $\mathrm{O}_{2}, \mathrm{NO}$, and $\mathrm{N}_{2}$. In the reaction of $\mathrm{O}^{-}$with $\mathrm{CH}_{4}$ we have shown that stretching vibrations increase the reactivity while bending vibrations do not.

When the NOAA group designed their flowing afterglow to reach $900 \mathrm{~K}$, they stated that " $900 \mathrm{~K}$ is the practical limit for a flowing afterglow system." ${ }^{6}$ We have circumvented this limit by building a flowing afterglow apparatus from scratch to operate only at room temperature and above. A much simpler design results from relaxing the constraint that the system must also be cooled to operate below room temperature.

\section{ACKNOWLEDGMENTS}

The authors would like to thank Arthur Fontijn and Dave McFadden for helpful discussions. The instrument was funded in part by the Air Force Office of Scientific Research.

${ }^{1}$ J. M. Farrar and W. H. Saunders, Jr., Techniques for the Study of IonMolecule Reactions (Wiley, New York, 1988).

${ }^{2}$ A. A. Viggiano, R. A. Morris, and J. F. Paulson, Int. J. Mass Spectrom. Ion Processes 135, 31 (1994).

${ }^{3}$ R. A. Morris and A. A. Viggiano, J. Phys. Chem. 98, 3740 (1994).

${ }^{4}$ Y. Ikezoe, S. Matsuoka, M. Takebe, and A. A. Viggiano, Gas Phase Ion-Molecule Reaction Rate Constants Through 1986 (Maruzen, Tokyo, 1987).

${ }^{5}$ W. Lindinger, F. C. Fehsenfeld, A. L. Schmeltekopf, and E. E. Ferguson, J. Geophys. Res. 79, 4753 (1974).

${ }^{6}$ E. E. Ferguson, J. Am. Soc. Mass Spectrom. 3, 479 (1992).

${ }^{7}$ A. Chen, R. Johnsen, and M. A. Biondi, J. Chem. Phys. 69, 2688 (1978).

${ }^{8}$ A. Fontijn and P. M. Futerko, in Gas Phase Metal Reactions, edited by A. Fontijn (Elsevier Science, Amsterdam, 1992), p. 93.

${ }^{9}$ K. Hilpert, in Structure and Bonding, edited by M. J. Clarke, J. B. Goodenough, J. A. Ibers, D. M. Jorgenson, D. M. Mingos, J. B. Neilands, G. A. Palmer, D. Reinen, P. J. Sadler, R. Weiss, and R. J. P. Williams (Springer, Berlin, 1990), p. 97.

${ }^{10}$ L. R. Watson, T. L. Thiem, R. A. Dressler, R. H. Salter, and E. Murad, J. Phys. Chem. 97, 5577 (1993).

${ }^{11}$ S. J. Burns, J. M. Matthews, and D. L. McFadden, J. Phys. Chem. (submitted).

${ }^{12}$ R. G. Levy, S. J. Burns, and D. L. McFadden, Chem. Phys. Lett. 231, 132 (1994).

${ }^{13}$ T. M. Miller, J. F. Friedman, M. Menendez-Barreto, A. A. Viggiano, R. A. Morris, A. E. S. Miller, and J. F. Paulson, Phys. Scr. T53, 84 (1994).

${ }^{14}$ E. E. Ferguson, F. C. Fehsenfeld, D. B. Dunkin, A. L. Schmeltekopf, and H. I. Schiff, Planet. Space Sci. 12, 1169 (1964).

${ }^{15}$ E. E. Ferguson, F. C. Fehsenfeld and A. L. Schmeltekopf, in Advances in 
Atomic and Molecular Physics, edited by D. R. Bates (Academic, New York, 1969).

${ }^{16}$ S. T. Graul and R. R. Squires, Mass Spectrom. Rev. 7, 263 (1988).

${ }^{17}$ Mellen Co., Penacook, NH.

${ }^{18}$ BriskHeat Mfg. Co., Columbus, OH, model BW051080.

${ }^{19}$ C. J. Howard, J. Phys. Chem. 83, 3 (1979).

${ }^{20}$ Eurotherm Controls, Reston, VA Model 818 digital control and model 831 SCR assembly. The thermocouples were "type $R$ " (Pt/Pt 13\% Rh) and "type $C$ " (W 5\% Re/W 26\% Re).

${ }^{21}$ Aremco Products Inc, Ossining, NY.

${ }^{22}$ M. Gilbert, Combust. Flame 2, 149 (1958).

${ }^{23}$ C. R. Hammond, in Handbook of Chemistry and Physics, edited by R. C. Weast (Chemical Rubber, Boca Raton, FL, 1975), p. B-18.
${ }^{24}$ A. A. Viggiano, J. M. Van Doren, R. A. Morris, and J. F. Paulson, J. Chem. Phys. 93, 4761 (1990).

${ }^{25}$ E. E. Ferguson, J. M. Van Doren, A. A. Viggiano, R. A. Morris, J. F. Paulson, J. D. Stewart, L. S. Sunderlin, and P. B. Armentrout, Int. J. Mass Spectrom. Ion. Processes 117, 261 (1992).

${ }^{26} \mathrm{~T}$. M. Miller (unpublished data taken from a flowing afterglow Langmuir probe apparatus).

${ }^{27}$ R. C. Bolden, R. S. Hemsworth, M. J. Shaw, and N. D. Twiddy, J. Phys. B 3, 45 (1970).

${ }^{28}$ Unpublished data.

${ }^{29}$ A. A. Viggiano, R. A. Morris, F. Dale, J. F. Paulson, K. Giles, D. Smith, and T. Su, J. Chem. Phys. 93, 1149 (1990). 